\section{Double cord blood transplantation: co-operation or competition?}

\author{
Nikolaos Neokleous, ${ }^{1}$ Anastasia Sideri, ${ }^{2}$ \\ Corina Peste-Tsilimidos ${ }^{2}$
}

${ }^{1}$ Hematological Clinic, General Hospital of Limassol, Cyprus; ${ }^{2}$ Stem Health Hellas S.A., Hygeia Hospital, Athens, Greece

\section{Abstract}

Over the last two decades umbilical cord blood (UCB) transplantation (UCBT) is increasingly used for a variety of malignant and benign hematological and other diseases. The main factor that limits the use of UCB to low weight recipients, mainly children and adolescents, is its low progenitor cell content. Various alternatives have been exploited to overcome this difficulty, including the transplantation of two UCB units (double umbilical cord blood transplantation, dUCBT). Following dUCBT, donor(s) hematopoietic stem cells (HSC) can be detected in the peripheral blood of the recipient as soon as 14 days post-transplantation. Sustained engraftment of HSC from one or both donors can be observed- dominance or mixed chimerism respectively, although single donor unit dominance has been observed in over $85 \%$ of patients. The underlying biology, which accounts for the interactions both between the two infused UCB units- cooperative or competitive, and with the recipient's immune system, has not been elucidated.

\section{Brief Report}

Since the first dUCBT in 2005 , its safety and efficacy have been examined in over 993 patients.1 Compared to single UCB, dUCBT is associated with: i) Higher incidence of acute GvHD grade II, though not higher treatmentrelated mortality or chronic GvHD; ii) Lower leukemia relapse for patients with good disease status (complete remission $1-2$ ). ${ }^{1-3}$ In more than $85 \%$ of patients undergoing dUCBT, regardless of the conditioning scheme, longterm hematopoiesis is derived from one of the infused cord blood units. ${ }^{1,4}$ The time-frame for the engraftment of the dominant unit has not yet been elucidated. However, in over $80 \%$ of patients, single unit dominance can be detected three weeks post-transplantation. ${ }^{4}$ Mixed chimerism can be detected in the one fifth of the patients under reduced intensity conditioning (RIC) regimens4. Cases whereby dom- inance reversion or loss of single unit dominance in favor of mixed donor chimerism have, also, been reported. ${ }^{5,6}$ There are mathematical models that can provide approximations of the chimerism pattern following dUCBT, but the prediction of the winning unit seems to be impossible (atmospheric noise theory) ${ }^{7,8}$

In attempting to explain single unit dominance in dUCBT, both intrinsic properties of the infused units and immune interactions between the recipient and the donors are taken into consideration. However, the former are difficult to rationalize, especially since variations regarding the in vitro proliferation potential of UCB CD34+ cells have been reported. ${ }^{9}$ Nevertheless, it has been demonstrated that there is no association between dominance and number of nucleated cells, CD34+, $\mathrm{CD}^{+}$, degree of HLA/sex mismatch, ABO group, viability, order and route of infusion. ${ }^{4}$ However, Avery et al reported an association between higher $\mathrm{CD}^{+}$cell dose and unit dominance in patients undergoing dUCBT following myeloablative regime. ${ }^{10}$ Cell viability is a controversial issue. Clinical experience shows that cord blood with viability less than $70 \%$ could be easily engrafted, although Scaradavou et al. recently analyzed 46 cord blood transplants and suggested that low CD34+ cell viability $(<75 \%)$ UCB units in dUCBT have low probability of engraftment. ${ }^{11}$ In this study, infusion of one high $(>75 \%)$ and one low (75\%) $\mathrm{CD}^{+} 4^{+}$viability unit resulted in engraftment of the high viability unit. Either unit engrafted in patients transplanted with two units of high (27 patients) or low viability (1 patient). It has, also, been proposed that the order of infusion may influence unit dominance. Intravenous infusion of the units in dUCBT with 3.5-4.5 hour interval promotes the engraftment of the first infused unit. ${ }^{5}$ Bearing in mind that the HSC could home to the endosteal niche in under five hours post-infusion, it is likely that even a short interval may contribute to the dominance of the first infused unit. ${ }^{12}$ Furthermore, the tight balance between proliferation and quiescence of the resident stem cells in the endosteal niche could influence the long-term engraftment of the dominant unit. ${ }^{13}$ Clinical trials comparing the different routes of infusion have not demonstrated any selective advantage between intravenous and intrabone administration. ${ }^{14}$

On the other hand, there is increasing evidence that single unit dominance in dUCBT recipients is the result of the immune-mediated rejection of the non-engrafting unit. ${ }^{5}$ It has been demonstrated in vivo that naive $\mathrm{CD}^{+} \mathrm{T}$ cells in one UCB unit expanded and differentiated into IFN $-\gamma$ secreting effector T cells that specifically recognized the non-engrafting unit and caused its rejection. ${ }^{9}$ However, these cytotoxic cells were transiently detected in the
Correspondence: Nikolaos Neokleous, Hematological Clinic General Hospital of Limassol, Cyprus.

E-mail: nneokleous@yahoo.gr

Key words: Cord blood transplantation, engraftment, dominance, chimerism.

Fundings: A.S. and C.P-T. are funded by STEM HEALTH-HELLAS, Hygeia Hospital.

Conflict of interest: the authors report no conflicts of interest.

Received for publication: 17 May 2011.

Revision received: 6 June 2011.

Accepted for publication: 10 June 2011.

This work is licensed under a Creative Commons Attribution NonCommercial 3.0 License (CC BYNC 3.0).

(C) Copyright N. Neokleous et al., 2011

Licensee PAGEPress, Italy

Hematology Reports 2011; 3:e6

doi:10.4081/hr.2011.e6

peripheral blood of dUCBT recipients with single unit dominance and are, therefore, not likely to be the sole cause of rejection. The chronic GvHD in patients with mixed chimerism following RIC regimens suggests graft-versus-graft interactions between the two units and between the units and the recipient. ${ }^{5}$ In cases with mixed chimerism, more studies are needed to clarify the interactions between the three different elements, the two infused units and the recipients. A recent study provides further evidence in favor of immune interactions between the infused units, since recipients of units closely (7-10 to 10-10) HLA-matched to each other, undergoing myeloablative regime, were more likely to demonstrate initial engraftment of both units. ${ }^{10}$

Bearing in mind the incompatibility between the two units, the allo-reactive response could be triggered by immune system components, such as minor $\mathrm{H}$ antigens that are shared between the UCB units. This hypothesis could account for the enhanced graft-versus-leukemia (GvL) effect associated with dUCBT, if the progenitor cells of the nonengrafted unit have similar major or minor antigens with the leukemic cells. It is not clear whether HLA disparity contributes, too. The identification of the antigens expressed on HSCs that activate the T-cells of the dominant unit is ongoing. Furthermore, the in utero development of $\mathrm{CD} 4{ }^{+} \mathrm{T}$ cells, which can be tolerant to non-inherited maternal allo-antigens present in the other UCB unit, could account for the mixed chimerism.9,15 Studies on murine models revealed that the addition of 
the corresponding mononuclear cells or CD34to $\mathrm{CD} 34^{+}$cells restored single-unit dominance following dUCBT, suggesting that unit dominance is probably associated with T-cell mediated graft-versus-graft immune interactions. Other immune-related mechanisms can, also, be involved, such as killer-immunoglobulinlike receptor-ligand incompatibility and NKcell activation. ${ }^{16,17}$

Several clinical trials have shown that cotransplantation of third-party mesenchymal stromal cells (MSCs) derived from various sources (bone marrow, placenta) could improve the engraftment, although marginally. ${ }^{18}$ In murine models, dUCBT accompanied by co-infusion of MSCs improved engraftment and reduced the extent of single unit dominance in favor of mixed chimerism. ${ }^{19}$ MSCs have immunosuppressive/immunomodulatory properties and exert trophic activity- via the secretion of immune-related molecules, with which they can modulate T-cell responses. ${ }^{19}$ Furthermore, culture-expanded MSCs do not express MHC class II surface markers and costimulatory molecules, so that they can neither function as antigen-presenting cells nor can they be directly involved in T-cell triggering. ${ }^{20}$ Whether the improved engraftment in the presence of MSCs is associated with improved homing or increased bone marrow tropism or promotion of immunotolerance remains to be determined.

In conclusion, intrinsic factors of the stem cells not yet fully understood, such as homing to the niche, as well as, prior therapy, intensity of conditioning regime, trophic effects, host factors and interactions between the grafts and the host are all likely contribute to the pattern of chimerism in dUCBT. The implication of immune-mediated mechanisms could be of significance in the context of leukemia; if the dominant unit can be predicted prior to transplantation; a non-engrafting unit sharing host antigens not present on the engrafting unit can be selected to promote the GvL effect. Ongoing clinical trials and prolonged patient follow-up will contribute in clarifying the underlying biology of dUCBT and demonstrate its safety and efficacy.

\section{References}

1. Rocha V, Crotta A, Ruggeri A, et al. Double cord blood transplantation: extending the use of unrelated umbilical cord blood cells for patients with hematological diseases. Best Pract Res Clin Haematol 2010;23:2239.

2. MacMillan ML, Weisdorf DJ, Brunstein CG, et al. Acute graft-versus-host disease after unrelated donor umbilical cord blood transplantation: analysis of risk factors. Blood 2009;113:2410-5.

3. Verneris MR, Brunstein CG, Barker J, et al. Relapse risk after umbilical cord blood transplantation: enhanced graft-versusleukemia effect in recipients of 2 units. Blood 2009;114:4293-9.

4. Delaney C, Gutman JA, Appelbaum FR. Cord blood transplantation for haematological malignancies: conditioning regimens, double cord transplant and infectious complications. $\mathrm{Br} \mathrm{J}$ Haematol 2009;147:207-16.

5. Haspel RL, Kao G, Yeap BY, et al. Preinfusion variables predict the predominant unit in the setting of reduced-intensity double cord blood transplantation. Bone Marrow Transplant 2008;41:523-9.

6. Yen HJ, Chiou TJ, Hung GY, et al. Longterm mixed full-donor chimerism with dominance reversion after a double-unit cord blood transplant. Eur J Haematol 2008;80:366-7.

7. Kristt D, Gesundheit B, Stein J, et al. Quantitative monitoring of multi-donor chimerism: a systematic, validated framework for routine analysis. Bone Marrow Transplant 2010;45:137-47.

8. Verneris MR, Wagner JE. UCB and atmospheric noise. Blood 2010;115:754-5.

9. Gutman JA, Turtle CJ, Manley TJ, Heimfeld Set al. Single-unit dominance after double-unit umbilical cord blood transplantation coincides with a specific CD8+ T-cell response against the nonengrafted unit. Blood. 2010;115:757-65.

10. Avery S, Shi W, Lubin M, et al. Influence of infused cell dose and HLA-match on engraftment after double-unit cord blood allografts. Blood 2010;117:3277-85.

11. Scaradavou A, Smith KM, Hawke R, et al. Cord blood units with low CD34+ cell viability have a low probability of engraftment after double unit transplantation. Biol Blood Marrow Transplant 2010;16:500-8.

12. Nilsson SK, Johnston HM, Coverdale JA. Spatial localization of transplanted hemopoietic stem cells: inferences for the localization of stem cell niches. Blood 2001; 97:2293-9.

13. Calvi LM, Adams GB, Weibrecht KW, et al. Osteoblastic cells regulate the haematopoietic stem cell niche. Nature 2003;425:841-6.

14. Brunstein CG, Barker JN, Weisdorf DJ, et al. Intra-BM injection to enhance engraftment after myeloablative umbilical cord blood transplantation with two partially HLA-matched units. Bone Marrow Transplant 2009;43:935-40.

15. Mold JE, Michaëlsson J, Burt TD, et al. Maternal alloantigens promote the development of tolerogenic fetal regulatory $\mathrm{T}$ cells in utero. Science 2008;322:1562-5.

16. Eldjerou LK, Chaudhury S, Baisre-de Leon A, et al. An in vivo model of double-unit cord blood transplantation that correlates with clinical engraftment. Blood 2010; 116:3999-4006.

17. Willemze R, Rodrigues CA, Labopin M, et al. KIR-ligand incompatibility in the graftversus-host direction improves outcomes after umbilical cord blood transplantation for acute leukemia. Leukemia 2009;23: 492-500.

18. Kim DW, Chung YJ, Kim TG, et al. Cotransplantation of third-party mesenchymal stromal cells can alleviate single-donor predominance and increase engraftment from double cord transplantation. Blood 2004;103:1941-8.

19. Caplan AI. Why are MSCs therapeutic? New data: new insight. J Pathol 2009;217: 318-24.

20. Le Blanc K, Tammik C, Rosendahl K, et al. HLA expression and immunologic properties of differentiated and undifferentiated mesenchymal stem cells. Exp Hematol 2003;31:890-6. 